\title{
Rapid Response System in the Recognising of the Deteriorating Patients: Respiratory Complaints are the Origins
}

\author{
Jaber Saud Alqahtani \\ Advanced Clinical Practice (Critical Care) Program, University of Southampton, School of Health Sciences, Southampton, United Kingdom
}

*Corresponding author: Jaber Saud Alqahtani, Advanced Clinical Practice Citation: Alqahtani JS (2016) Rapid Response System in the Recognising of (Critical Care) Program, University of Southampton, School of Health the Deteriorating Patients: Respiratory Complaints are the Origins. Enliven: J Sciences, Southampton, United Kingdom, E-mail: J1aa@hotmail.com Anesthesiol Crit Care Med 3(2): 004.

Received Date: $02^{\text {nd }}$ June 2016 Accepted Date: $13^{\text {th }}$ July 2016

Published Date: $18^{\text {th }}$ July 2016

Copyright: 2016 MR. Jaber Saud Alqahtani. This is an Open Access article published and distributed under the terms of the Creative Commons Attribution License, which permits unrestricted use, distribution and reproduction in any medium, provided the original author and source are credited.

\begin{abstract}
Rapid response team (RRT) system is a structure that contains a set of clinical criteria for certain conditions to detect and intervene on any abnormal signs and symptoms that do not meet these criteria by a qualified expert team [1]. To date, as a professional respiratory therapist in Saudi Arabia, one of the most significant challenges for the Ministry of Health is to provide high standards of professional quality without harm. Notable examples of such a challenge are increasing numbers of patients with special care needs and a lack of a comprehensive team able to detect and manage acute symptoms of deteriorating patients before an emergency arises, and that specifically in wards areas. This is exemplified in a Saudi Arabian study conducted by Rehmani et al.[2] showing around $82 \%$ of ward patients who had cardiac arrests had deterioration signs in the previous 24 hours. Recently, several papers have been published reporting positive effects after implementations of RRT. Therefore, this article will review the literature in light of recent evidence to determine the implementation effectiveness of RRT system and role of respiratory therapists in improving serious conditions at the appropriate time for patients in hospital wards and decrease number of deaths.
\end{abstract}

Keywords: Rapid response team; Respiratory therapist; Cardiopulmonary arrest; Patient deterioration; Critical care

\section{Background}

According to Naeem and Montenegro [3] most cardiopulmonary arrests in hospitals are preceded by various warning signs. These warning signs are usually physiological abnormalities and signs of deterioration that are either not detected or not treated adequately. In 1999 the report, To Err Is Human: Building a Safer Health System was conducted [4]. This report describes several serious clinical health issues related to human error. It seeks solutions and initiatives from health care partners, including the workers, medical care governors, consumers and policy makers. The Institute of Medicine reported that around 98,000 patients died as a result of human errors [5]. As this report was seeking initiatives, the Institute for Healthcare Improvement announced an initiative that was recognised as The 100,000 Lives Campaign. It recommends six methods to reduce the mortality rate of inpatients in the United Sates by 100,000 between 2004 and 2006. A rapid response team (RRT) was one of the recommended systems to be implemented across health care facilities [6]. According to Ranji et al. [7], in response to this initiative many health care facilities have since the RRT system across the world.
Deterioration of patients and sudden respiratory and cardiac arrest in hospital wards can be avoided through the anticipation of suspected cases, which can be achieved by introducing a swift response strategy: cross disciplinary team strategy [8]. The right response at the right time when assessing patients who are deteriorating is crucial. Such a response should be undertaken by an expert team that is highly supported by new initiatives since a deterioration of patients outside ICU areas have profound health consequences for all patients of all ages [9]. According to Jones and Bellomo [10] in Europe, Australia and the USA, there are several studies stating that the deterioration of hospitalised patients in areas outside ICUs could be linked to the health systems that failed to respond early. Esmonde et al. [11] argue that a lack of ability to provide quick and efficient medical care to patients whose conditions are deteriorating is a significant, avoidable and a noticeable mistake. 
In the literature, it has been demonstrated that nearly $50 \%$ of respiratory and cardiac arrests cases are headed by signs and symptoms of deterioration 6 to 8 hours prior to arrest [12-15]. Usually, codes are used in hospitals for medical practitioners, for example the 'code blue team' indicates serious emergency cases [14]. Indeed, 'Code blue' is the most popular code used to indicate urgent resuscitation, mostly as an outcome of respiratory or cardiac arrest [15].

However, a clear benefit of code blue in the prevention of respiratory and cardiac arrest could not be identified because the team effectively arrives too late at the point of, or after, the arrest. The most surprising aspect of the code blue team is that despite being in hospitals for years, there remains no significant rise in the success rate, as its rate is below $15 \%$ [16]. In fact, the death rate is gradually increasing as a result of unexpected patient deterioration and cardiopulmonary arrests [17]. The development of awareness to unforeseen medical deterioration and the increase number of respiratory and cardiac arrests promote the necessity to implement RRT. Such a system has several aspects that differ from the code blue team and these aspects will provide a great opportunity to avoid cardiopulmonary arrests (Table 1) [18].

\begin{tabular}{|l|l|l|}
\hline Feature & Code blue team & Rapid response team \\
\hline Typical criteria for calling the team & $\begin{array}{l}\text { No recordable pulse, no recordable blood } \\
\text { pressure, absence of respiratory effort, } \\
\text { unresponsive }\end{array}$ & $\begin{array}{l}\text { Low blood pressure, rapid heart rate, respi- } \\
\text { ratory distress, altered consciousness }\end{array}$ \\
\hline Typical conditions that the team assesses and treats & $\begin{array}{l}\text { Cardiac arrest, respiratory arrest, airway } \\
\text { obstruction }\end{array}$ & $\begin{array}{l}\text { Sepsis, pulmonary edema, arrhythmias, } \\
\text { respiratory failure }\end{array}$ \\
\hline Typical team composition & $\begin{array}{l}\text { Anesthesia fellow, ICU fellow, internal } \\
\text { medicine house staff, ICU nurse }\end{array}$ & $\begin{array}{l}\text { ICU fellow, ICU nurse, respiratory therapist, } \\
\text { internal-medicine house staff }\end{array}$ \\
\hline Typical call rate (no./1000 admissions) & $0.5-5$ & $20-40$ \\
\hline Typical in-hospital mortality (\%) & $70-90$ & $0-20$ \\
\hline
\end{tabular}

Table 1 Comparison between a Code Blue Team and a Rapid-Response Team

\section{The Role of RRT}

RRT has several titles in the literature that are used interchangeably, including medical emergency teams (METs), patient-at-risk teams (PARTs), medical emergency response teams (MERTs) and critical care outreach teams (CCOTs) [19]. This team has been recognised as a system that contains a set of clinical criteria for certain conditions to detect and intervene on any abnormal signs and symptoms that do not meet these criteria by a qualified expert team [1]. RRT is a multidisciplinary team that compromises various clinical staff, including a physician, a respiratory therapist and a nurse. This team is principally responsible for the evaluation of patients who have signs and symptoms that indicate deterioration in hospital ward areas [5].

In fact, rapid response systems have various arrangements, as there is no ideal model and this could be related to the lack of comparative data among these models in the literature. According to Ranji et al. [7], there are two RRT models which are team is leading by doctors with nurses and team is leading by nurses or other critical care staff. This system has two major components, which are afferent and efferent limbs [1]. An afferent limb is responsible for identifying signs of deterioration in patients according to predefined criteria to activate an RRT response. Whereas an efferent team is to respond quickly by attending the intervention to assess patients. Despite these different types, the presence of a respiratory therapist is crucial, in my view. The importance of having a respiratory therapist in the RRT is because clinical conditions that progress to be more serious are often breathing problems. Moreover, in some cases, if the main problem is not respiratory, at least one part of the respiratory function will be affected and be a major part of the clinical issue [20]. Based on my experience, respiratory complications are a strong risk factor for the deterioration of patients that ultimately lead to cardiopulmonary arrest.

\section{Respiratory Conditions are the Origins}

Respiratory problems usually have a substantial role in developing cardiac arrhythmias such as pulse less electrical activity (PEA) and a systole. Furthermore, the common reasons of PEA consist of PH acidosis (respiratory from decreased ventilation or metabolic), pneumothorax, reduction of $\mathrm{O} 2$ level in the tissue (hypoxia), fluid reduction and pulmonary embolus [21]. According to Schein et al. [22] before cardiac arrest, around $70 \%$ of the deterioration for patients was respiratory and these abnormalities can be predicted and prevented. Most of these causes are purely breathing conditions that have obvious symptoms. Moreover, there are physiological criteria generally utilised for calling RRT and these parameters are mostly breathing in nature. These elements are tachypnea, shortness of breath (SOB), respiratory distress, hypoxia, tachycardia and bradycardia, high blood pressure, chest pain and mental health change[18,23]. These signs and symptoms of deterioration illustrate that respiratory therapists have distinct functions in detecting and treating these cases than other members in the RRT.

Respiratory therapist can play a major role in both the afferent and efferent limbs of rapid response systems (RRS). In the afferent team, the respiratory therapist can support the nurses to identify abnormal signs and symptoms early that relate to respiratory and take action. In table 2 some respiratory physiological indicators are displayed for ventilation support [24]. Although these indictors can help in the decision to start ventilation support, patient status is still the most significant indicator. The purpose of ventilator support is to correct the oxygenation and ventilation status that could lead to respiratory failure [24]. Treating respiratory failure in the early stages will prevent further life threatening complications that lead to increased morbidity and mortality such as organ failure, sepsis and acute respiratory distress syndrome [25]. 
Table 2 Physiologic Indicators for Ventilatory Support

\begin{tabular}{|c|c|c|}
\hline Mechanism & Normal Values & Support Indicated \\
\hline $\begin{array}{l}\text { Inadequate Alveolar Ventilation } \\
\mathrm{PaCO}_{2}(\mathrm{~mm} \mathrm{Hg}) \\
\mathrm{pH}\end{array}$ & $\begin{array}{l}35-45 \\
7.35-7.45\end{array}$ & $\begin{array}{l}>55 \\
<7.20\end{array}$ \\
\hline \multicolumn{3}{|l|}{ Inadequate Lung Expansion } \\
\hline $\begin{array}{l}\text { Tidal volume }(\mathrm{VT}) \mathrm{ml} / \mathrm{kg} \\
\text { Vital capacity }(\mathrm{VC}) \mathrm{ml} / \mathrm{kg} \\
\text { Respiratory rate }\end{array}$ & $\begin{array}{l}5-8 \\
65-75 \\
12-20\end{array}$ & $\begin{array}{l}<5 \\
<10 \\
>35 \text { or }<10\end{array}$ \\
\hline $\begin{array}{l}\text { Inadequate Muscle Strength } \\
\text { Maximum inspiratory pressure }\left(\mathrm{cm} \mathrm{H}_{2} \mathrm{O}\right) \\
\text { Vital capacity }(\mathrm{VC}, \mathrm{ml} / \mathrm{kg}) \\
\text { Maximum voluntary ventilation }(\mathrm{MVV}, \mathrm{L} / \mathrm{min})\end{array}$ & $\begin{array}{l}-80-100 \\
65-75 \\
120-180\end{array}$ & $\begin{array}{l}\geq-20 \\
<10 \\
<2 * \operatorname{VE}(\text { minute ventilation) }\end{array}$ \\
\hline $\begin{array}{l}\text { Increased Work of Breathing } \\
\text { Minute ventilation }(\mathrm{V} \neg \mathrm{E}) \mathrm{L} / \mathrm{min} \\
\mathrm{VD} / \mathrm{VT}(\%)\end{array}$ & $\begin{array}{l}5-6 \\
0.25-0.40\end{array}$ & $\begin{array}{l}>10 \\
>0.6\end{array}$ \\
\hline \multicolumn{3}{|l|}{ Hypoxemia } \\
\hline $\begin{array}{l}\mathrm{P}(\mathrm{A}-\mathrm{a}) \mathrm{O}_{2} \text { on } 100 \% \mathrm{O}_{2}(\mathrm{~mm} \mathrm{Hg})>350 \\
\mathrm{PaO}_{2} / \mathrm{FiO}_{2}\end{array}$ & $\begin{array}{l}25-65 \\
350-450\end{array}$ & $\begin{array}{l}>350 \\
<200\end{array}$ \\
\hline
\end{tabular}

Moreover, the respiratory therapist can provide support for several conditions including, but not limited to, chronic obstructive pulmonary disease (COPD), pulmonary edema, acute asthma and acute lung injury [24]. Non-invasive ventilation (NIV), suctioning and bronchial hygiene protocols can be used by the respiratory therapist to decrease the work of breathing and relieve COPD patients, and this action also prevents further deterioration [26,27] Furthermore, according to Gray [28], using NIV with pulmonary edema is recommended to correct the respiratory failure and prevent the need for intubation. What is clear from the above is that roles of a respiratory therapist in the afferent team are important and will help in detecting and preventing further deterioration in respiratory conditions.

On the other hand, the respiratory therapist can have an essential role in the efferent team, for example; protect the airway, resuscitation and respiratory intervention [1]. In this team, the respiratory therapist can support patients by using invasive mechanical ventilation if NIV failed to protect the airway and correct the hypoxemia. Based on my experience, respiratory therapists can support in transferring and monitoring the patients in the ICU by using a ventilator sheet every 4 hours to maintain a high standard care and prevent further deterioration. However, the cooperation of the respiratory therapist and other members in the team, especially the nurses, will have remarkable effects in reducing the respiratory and cardiac arrests and ultimately mortality rate.

\section{Prioritisation and Callout Time}

Patients' conditions must be the top priorities of health care practitioners. Conditions that patients have are varied and need efficient skills and knowledge in order to differentiate and prioritise [29]. According to Lake et al. [30] prioritisation involves the selection of necessities among choices. Failure to identify cases that need immediate action against those that require routine care is a danger and could cause further deterioration in urgent cases [30]. Therefore, RRT was created to detect abnormal signs and symptoms that happen prior to cardiac arrest. However, the callout time is a significant part in the decision-making process. Indeed, the callout occurs when the responsible and trained staff detect and recognise the warning signs that cause patient distress and call the RRT for further investigation [31]
The decision to activate RRT is based on either objective or subjective information; examples of objective data are abnormal (respiratory rate, heart rate, oxygen saturation, blood pressure). On the other hand, subjective data depends on the feelings and concerns of the nurse about their patient. According to Cioffi [32] nurses use their experiences and intuition to activate RRT. Furthermore, another study revealed that nurses were using both subjective and objective data; however, their decisions relied more on subjective data [33]. These studies indicate the significance of the experience while taking decisions. However, Nyatanga and Vocht [34] argue that intuition is not evidence based and this commonly leads to errors in judgment [32]. It has been suggested that our decisions should be a combination of both scientific information and intuition in order to have optimal results [35]. Therefore, experience, knowledge and evidence based practice are maximally required in the decisions of the RRTs either in the afferent or efferent team for an efficient overall system.

\section{Effectiveness of RRT in clinical sites and its challenges}

Health care institutions have conducted numerous papers to assess RRT effectiveness on the quality of care in clinical sites. These institutions targeted specific clinical outcomes including unplanned ICU admission, cardiopulmonary arrests rate, rate of mortality and hospital inpatient days.

Regarding the cardiopulmonary arrests rate, one study documented that after the implementation of RRT, the overall hospital code rates reduced from 11.2 to 7.5 per 1,000 patient admissions [14]. However, there was no significant effect on the mortality rate. Similarly, another study by Goncales et al. [36] found that the number of cardiopulmonary arrests significantly decreased from 3.54 to 1.69 per 1,000 patient discharges as well as the rate of mortality from 16.30 to 14.40 per 1,000 patient admissions [36]. In Saudi Arabia, there was one study in 2012 that showed a similar result with previous studies. They revealed that around 75 cases were documented as cardiopulmonary arrests in 2006 before RRT was implemented, and this number decreased by around $50 \%$ at 37 cardiopulmonary arrests after they introduced RRT [37]. Furthermore, Baxter et al. [38] identified very important outcomes when comparing before and after RRT implementation. Cardiac arrests decreased 
from 2.53 to 1.5 per 1,000 admissions, admissions to the ICU fell from 42.3 to 37.6 and finally readmissions after ICU discharge fell from 13.5 to 8.8 . Also, they conclude that successful implementation of RRT has a significant impact on reducing morbidity and cost of ICUs [38]. By contrast, a number of studies have investigated the effects of RRT pre and post implementation and found no significant changes or considerable effects on clinical outcomes [39-41].

Additionally, the efficiency of RRT on certain kinds of patients was assessed by utilising prospective design research. Konrad et al. [41] highlight the rate of mortality of ward patients that declined by $12 \%$ and also surgical patients, reduced by $28 \%$ which lead to a decrease in the cardiopulmonary arrests rate of both types from 1.12 to 0.74 . Whereas some studies have concentrated on the relation between the rise in the number of calls of RRT and the decline in deterioration factors. This is exemplified in the work undertaken by Devita et al. [1] that point out clear relations between these two factors as the outcomes from a retrospective investigation that revealed a significant decline of $17 \%$ in the cardiopulmonary arrest rate as a result of increasing RRT calls from afferent teams. In the same vein, Dacey et al. [42] and Wood et al. [43] have reported similar outcomes. However, the aforementioned outcomes were not only assessed in the literature, other clinical outcomes have been investigated across several studies. ICU admissions and readmissions were evaluated by Garcea et al. [44] and McFarlan and Hensley [45] who found that comprehensive and proper management of deteriorating patients outside the ICU areas dramatically decrease the need for ICU admission. Also, readmissions to ICU declined after frequent follow ups with the patients that transferred to the wards from the ICU.

In terms of study designs, various systematic reviews have been developed and introduced to point out the weakness points of many trials that assess the effectiveness of RRT $[5,7,8]$. These reviews highlight some limitations for example, limited to one hospital, short periods of RRT observation, retrospective studies and different RRT training. These factors could impact the reliability and generalisability of results. Similarly, in the review carried out by Tee et al. [16] although the advantages of RRT implementation are readily recognisable, most studies that have methodological limitations result in a low quality of evidence which cannot change the clinical practice or improve the care quality. In addition, the management of health care facilities, particularly the policies and protocols, can constrain RRT implementation, which ultimately alters the real outcomes [46]. Likewise, Ranji et al. [7] conducted a systematic review and meta-analysis that reported differences in RRT members in hospitals will affect the accuracy of results. For example, some RRTs are led by different hospital staff, for instance doctors or nurses or respiratory therapists, and this will lead to variations in outcomes.

In 2004, Priestley et al. [47] conducted the first RCT in the UK investigating the impact of implementing an RRT service on the hospital wards and length of stay in hospital. They found that RRT systems decrease the rate of mortality in hospital wards overall, compared to control wards. However, they documented that duration of stay could be increased with this system and it is still debatable as they were unable to confirm from their analysis. A serious weakness with this RCT, however, is that the short period of observation was 60 days, and the randomisation caused concern because control and intervention groups were included $[5,8]$
Furthermore, another multicentre RCT was published in 2005 which found no significant effects and no relations among the incidence of cardiopulmonary arrests, sudden ICU admissions, unanticipated deaths and RRT implementation [40]. However, one criticism across much of the literature on this trial is that there was no standardisation during the RRT implementation in the sites that participated, inadequate education during this period and the time period for this investigation, which was only 6 months, where such a system needs at least two years to be evaluated $[7,46]$.

Recently, one systematic review and meta-analysis was conducted in 2016 to evaluate the RRT effectiveness on hospital mortality and cardiopulmonary arrests [48]. They conclude that RRT implementation has reduced both hospital mortality and cardiopulmonary arrests.

In summary, it is worth noting that despite the challenges and limitations for the RRT implementation, such as a shortage of RCTs, short time periods and different levels of training, RRT system implementation has a wealth of positive aspects to improve the quality of care.

\section{Recommendations for Future Implementation of RRT System}

Evaluation of such a system requires rigorous research designs to ensure reliable and convincing data. Despite the ethical challenges that can constrain RCTs in clinical practice, large international multicentre RCTs are needed to assess important clinical outcomes such as cardiac arrests reduction, morbidity and mortality reduction and unplanned admission. Such studies should be carried out internationally to ensure reproducibility of their results. Furthermore, qualitative studies may also provide a good exploration of contextual factors that influence RRT systems. Additionally, RRTs should include various experts from a number of clinical backgrounds and this will help in diagnosing and treating cases promptly. In Saudi Arabia, some hospitals with RRT systems do not include respiratory therapists; however, the respiratory therapist has an important role in detecting and treating clinical problems that are purely respiratory. Moreover, it is important to utilise simulation in educating the RRT members as suggested by Leonard et al. [49]. This tool can improve and enhance the confidence and performance for members of RRTs as are exposed to various conditions in practice to build and improve their clinical decisions. Finally, RRTs should improve the quality of care, as Hijasi et al.[37] found; around 30\% of the responses of RRT are a result of medical human error, therefore reporting these errors to the management will help to raise awareness and decrease such margins.

\section{Conclusion}

To conclude, an RRT system has several important advantages resulting in a noteworthy decline in the frequency of cardiopulmonary arrests, and in most areas, the rate of mortality will be decreased following developments in the quality of care. RRT system can save patients from deterioration, as most signs and symptoms start a long enough time prior to severe deterioration onset when such events need immediate action. Furthermore, RRT system tends to change the health care culture and this change will lead to inform good by improving the critical thinking of staff and the decision making processes leading to better patient outcomes. As most of the cases that deteriorated are from respiratory compromise, respiratory 
therapists have significant value in the management. Finally, implementation of RRT system in Saudi Arabia is highly recommended and the role of the respiratory therapist should be a central with other roles in the RRT system to upgrade clinical practice and meet the patient and institution expectations.

\section{Formatting of Funding Sources}

This research did not receive any specific grant from funding agencies in the public, commercial, or not-for-profit sectors.

\section{References}

1) Devita MA, Bellomo R, Hillman K, Kellum J, Rotondi A, et al. (2006) Findings of the first consensus conference on medical emergency teams. Crit Care Med 34: 2463-2478.

2) Rehmani R, Memon JI, Nizam IY (2009) Warning signs prior to inhospital cardiac arrest. Need for a rapid response team. Saudi Med J 30: 580-582.

3) Naeem N, Montenegro H (2005) Beyond the intensive care unit: A review of interventions aimed at anticipating and preventing in-hospital cardiopulmonary arrest. Resuscitation 67: 13-23.

4) Kohn LT, Corrigan JM, Donaldson MS (2000) To err is human: building a safer health system. Ann Fr Anesth Reanim.

5) Sakai T, DeVita MA (2009) Rapid response system. J Anesth 23: 403408.

6) Berwick DM, Calkins DR, McCannon CJ, Hackbarth AD (2006) The 100,000 lives campaign: setting a goal and a deadline for improving health care quality. JAMA 295: 324-327.

7) Ranji SR, Auerbach AD, Hurd CJ, O’Rourke K, Shojania KG (2007) Effects of rapid response systems on clinical outcomes: Systematic review and meta-analysis. J Hosp Med 422-432.

8) McGaughey J, Alderdice F, Fowler R, Kapila A, Mayhew A, et al. (2007) Outreach and Early Warning Systems (EWS) for the prevention of intensive care admission and death of critically ill adult patients on general hospital wards. Cochrane database Syst Rev CD005529.

9) Winters BD, Weaver SJ, Pfoh ER, Yang T, Cuong J, et al. (2013) Rapidresponse systems as a patient safety strategy: A systematic review. Ann Intern Med 158: 417-425.

10) Jones $D$, Bellomo R (2006) Introduction of a rapid response system: why we are glad we MET. Crit Care 10: 121.

11) Esmonde L, McDonnell A, Ball C, Waskett C, Morgan R, et al. (2006) Investigating the effectiveness of critical care outreach services: A systematic review. Intensive Care Med 32: 1713-1721.

12) Buist MD, Jarmolowski E, Burton PR, Bernard SA, Waxman BP, et al. (1999) Recognising clinical instability in hospital patients before cardiac arrest or unplanned admission to intensive care . A pilot study in a teriary-care hospital. Med J Aust 171: 22-25.

13) Bedell SE, Deitz DC, Leeman D, Delbanco TL (1991) Incidence and characteristics of preventable iatrogenic cardiac arrests. JAMA 265: 2815-2820.

14) Chan PS, Khalid A, Longmore LS, Berg RA, Kosiborod M, et al. (2008) Hospital-wide code rates and mortality before and after implementation of a rapid response team. JAMA 300: 2506-2513.
15) Sharek PJ, Parast LM, Leong K, Coombs J, Earnest K, et al. (2007) Effect of a rapid response team on hospital-wide mortality and code rates outside the ICU in a Children's Hospital. JAMA 298: 2267-2274.

16) Tee A, Calzavacca P, Licari E, Goldsmith D, Bellomo R (2008) Benchto-bedside review: The MET syndrome--the challenges of researching and adopting medical emergency teams. Crit Care 12: 205.

17) Adelstein BA, Piza MA, Nayyar V, Mudaliar Y, Klineberg PL, et al. (2011) Rapid response systems: a prospective study of response times. J Crit Care 26: 635.e11-e18.

18) Jones DA, DeVita MA, Bellomo R (2011) Rapid-Response Teams. N Engl J Med 365: 139-146.

19) Cretikos M, Parr M, Hillman K, Bishop G, Brown D, et al. (2006) Guidelines for the uniform reporting of data for Medical Emergency Teams. Resuscitation 68: 11-25.

20) Higgins Y, Maries-Tillott C, Quinton S, Richmond J (2008) Promoting patient safety using an early warning scoring system. Nurs Stand 22: 3540.

21) Neumar RW, Otto CW, Link MS, Kronick SL, Shuster M, et al. (2010) Part 8: Adult advanced cardiovascular life support: 2010 American Heart Association Guidelines for Cardiopulmonary Resuscitation and Emergency Cardiovascular Care. Circulation 122: S729-767.

22) Schein RM, Hazday N, Pena M, Ruben BH, Sprung CL (1990) Clinical antecedents to in-hospital cardiopulmonary arrest. Chest 98: 1388-1392.

23) Morgan RJM (1997) An early warning scoring system for detecting developing critical illness. Clin Intensive Care 8: 100.

24) Kacmarek RM (2016) Egan's Fundamentals of Respiratory Care. Elsevier - Health Sciences Division.

25) Hudson LD (1989) Survival data in patients with acute and chronic lung disease requiring mechanical ventilation. Am Rev Respir Dis 140: S19-S24.

26) McEvoy RD, Pierce RJ, Hillman D, Esterman A, Ellis EE, et al. (2009) Nocturnal non-invasive nasal ventilation in stable hypercapnic COPD: a randomised controlled trial. Thorax 64 : 561-566.

27) Wehrman S (2015) Workbook for Egan's fundamentals of respiratory care. St Louis, Mo Elsevier/Mosby.

28) Gray A, Goodacre S, Newby DE, Masson M, Sampson F, et al. (2008) Noninvasive Ventilation in Acute Cardiogenic Pulmonary Edema. N Engl J Med 359: 142-151.

29) Castledine G (2002) Prioritizing care is an essential nursing skill. Br J Nurs 11: 987-987.

30) Lake S, Moss C, Duke J (2009) Nursing prioritization of the patient need for care: A tacit knowledge embedded in the clinical decision-making literature. Int J Nurs Pract 15: 376-388.

31) Jamieson E, Ferrell C, Rutledge DN (2008) Medical emergency team implementation: experiences of a mentor hospital. Medsurg Nurs 17: 312-316, 323.

32) Cioffi J (2000) Nurses' experiences of making decisions to call emergency assistance to their patients. J Adv Nurs 32: 108-114.

33) Leach LS, Mayo A, O’Rourke M (2010) How RNs rescue patients: a qualitative study of RNs' perceived involvement in rapid response teams. Qual Saf Health Care 19: e13. 
34) Nyatanga B, Vocht H De (2008) Intuition in clinical decision-making: a psychological penumbra. Int J Palliat Nurs 14: 492-496.

35) D TC (2000) Decision making and judgement in nursing - an introduction in Thompson C and Dowding D (eds) Clinical Decision Making and Judgement in Nursing. Edinburgh: Churchill Livingstone: 1-20. In: D TC and D, editor. Clin Decis Mak Judgement Nurs (eds) Edinburgh: Churchill Livingstone; 1-20.

36) Goncales PD, Polessi JA, Bass LM, Santos GDe P, Yokota PK, et al. (2012) Fernandes Junior C, Cendoroglo Neto M, Estanislao M, Teich V, Sardenberg C. Reduced frequency of cardiopulmonary arrests by rapid response teams. Einstein (Sao Paulo) 10: 442-448.

37) Hijazi M, Sinno M, Alansar M(2012) The Rapid Response Team Reduces the Number of Cardiopulmonary Arrests and Hospital Mortality. Emerg Med 2: 128.

38) Baxter AD, Cardinal P, Hooper J, Patel R (2008) Medical emergency teams at The Ottawa Hospital: the first two years. Can J Anaesth 55: 223-231.

39) Bristow PJ, Hillman KM, Chey T, Daffurn K, Jacques TC, et al. (2000) Rates of in-hospital arrests, deaths and intensive care admissions: The effect of a medical emergency team. Med J Aust 173: 236-240.

40) Hillman K, Chen J, Cretikos M, Bellomo R, Brown D, et al. (2005) Introduction of the medical emergency team (MET) system: A clusterrandomised controlled trial. Lancet 365: 2091-2097.

41) Konrad D, Jaderling G, Bell M, Granath F, Ekbom A, et al. (2010) Martling CR. Reducing in-hospital cardiac arrests and hospital mortality by introducing a medical emergency team. Intensive Care Med 36: 100106.
42) Dacey MJ, Mirza ER, Wilcox V, Doherty M, Mello J, et al. (2007) The effect of a rapid response team on major clinical outcome measures in a community hospital. Crit Care Med 35: 2076-2082.

43) Wood KA, Ranji SR, Ide B, Dracup K (2009) Rapid response systems in adult academic medical centers. Jt Comm J Qual Patient Saf 35: 475482,437 .

44) Garcea G, Thomasset S, Mcclelland L, Leslie A, Berry DP (2004) Impact of a critical care outreach team on critical care readmissions and mortality. Acta Anaesthesiol Scand 48: 1096-1100.

45) McFarlan SJ, Hensley S (2007) Implementation and outcomes of a rapid response team. J Nurs Care Qual 22: 307-313, quiz 14-15.

46) Winters BD, Pham JC, Hunt EA, Guallar E, Berenholtz S, et al. (2007) Rapid response systems: a systematic review. Crit Care Med 35: 12381243.

47) Priestley G, Watson W, Rashidian A, Mozley C, Russell D, et al. (2004) Introducing Critical Care Outreach: a ward-randomised trial of phased introduction in a general hospital. Intensive Care Med 30: 1398-1404.

48) Solomon RS, Corwin GS, Barclay DC, Quddusi SF, Dannenberg MD (2016) Effectiveness of rapid response teams on rates of in-hospital cardiopulmonary arrest and mortality: A systematic review and metaanalysis. J Hosp Med 11: 438-445.

49) Leonard M, Graham S, Bonacum D (2004) The human factor: the critical importance of effective teamwork and communication in providing safe care. Qual Saf Health Care 13: i85-i90.

Submit your manuscript at

http://enlivenarchive.org/submit-manuscript.php

New initiative of Enliven Archive

Apart from providing HTML, PDF versions; we also provide video version and deposit the videos in about 15 freely accessible social network sites that promote videos which in turn will aid in rapid circulation of articles published with us. 